\section{Earthquake damage in Mexico City}

SIR-Flores et al. conclude from their study of resonance and earthquake damage in Mexico City that the surface distribution of damage within the soft-ground area can be explained by destructive and constructive interference of waves in the soft foundation material of the extinct lake'. During an extended visit to the area I was first struck by 'pockets of damage' scattered over the soft-ground region, and entertained similar ideas about interference of waves.

A detailed inspection of all buildings of more than five storeys, however, produced a far simpler explanation of the phenomenon. Tall buildings are not uniformly distributed over the Lago de Texcoco area of Mexico City but concentrated in regions like Roma Norte, Tlatelolco and Reforma. Predominantly these buildings suffered because of what may be called resonance ${ }^{2}$. Flores et al. also mention selective destruction of tall buildings. Buildings of few storeys generally suffered very little or no damage even if old and vulnerable. In the areas with low or no damage there were practically no tall buildings and therefore no structures likely to be damaged.

We do not deny the possibility of constructive or destructive interference. Earthquake damage is, however, controlled by many parameters ${ }^{3}$ and it is therefore very dangerous to rely on theoretical models only, especially if they are not supported by detailed observations. It must be remembered that earthquakes cause untold misery and inflict colossal financial losses. This is even more reason to compare carefully results produced by any model with the data derived from as large a sample as possible. This timeconsuming and costly sampling is dictated by the many damage parameters which must be considered. Some examples may serve to illustrate the potential pitfalls and the risk from future earthquakes if such rules are not heeded:

- The boundaries of the soft-ground region are not well known in the eastern region of the extinct lake. Any theoretical calculation is therefore bound to incorporate considerable uncertainties.

- The density of the soft material and therefore the wave velocity increases with depth. As the composition of the soft material is not homogeneous a very complex model and more precise information than available today would be needed to calculate interference patterns reliably.

- The heterogeneous soft material is excited by a very complex pattern of waves arriving from the rupture plane. Also this causes interference of waves in the soft material, adding to the many uncertainties.
- The comparatively few accelerographs did not give a uniform predominant period of $2 \mathrm{~s}$ but values ranging between $\sim 1.5$ and $3 \mathrm{~s}$. One may therefore wonder whether Flores et al. were right to select among the many solutions obtained only those which have the 'correct' frequency. - Hydrologists or meteorologists know that adding rain gauge data changes observed rainfall patterns. How reliable is the distribution of ground shaking derived from only a handful of instruments?

- Damage to buildings depends predominantly on: their structural strength; the effect of stiffening walls; architectural layout such as degree of irregularity of floor plan and of elevations (L-shape, Ushape, H-shape, T-shape); uniformity of distribution of masses, stiffness and damping; compatibility of building materials; orientational sensitivity; site effects (resonance). The importance of these parameters has been seen after many earthquakes ${ }^{4-8}$. Flores et al. have not considered these parameters in their conclusions.

Interesting as interference patterns may be, it appears to be dangerous to explain the damage pattern observed in Mexico City by the interference of waves in the soft ground.

Herbert Tiedemann

Swiss Reinsurance Co.

CH-8022, Zurich,

Switzerland

1. Flores, J., Novaro, O. \& Seliman, T.H. Nature 326, 783 785 (1987)

2. Tiedemann, H. Eighth Symp. Earthquake Engng 1, 23-30 (University of Roorkee, 1986).

Tiedemann. H. Engineering Geology 20, 169-179 (1984)

4. Imai, Ts. \& Okubo, T. Seventh World Conf. Earthquake Engineering Vol. 3, 33-40 (1980)

Marsal, R.J. Symp. Org. Nac de Est. Ing. Ingenieria 28(1), $10-23$ (1957).

6. Rosenblueth. E. Ingenieria XXXI(1), 1-31 (1961)

7. Shibuya, J., Kimura, H. \& Shiga. T. Seventh World Conf. Earthquake Engng Vol. 2. 199-206 (1980)

8. Tiedemann, H. Seventh World Conf. Earthquake Engng Vol. 2, 267-270 (1980); Vol. 6, 617-624 (1980); Seventh Eur. Conf. Earthquake Engng Vol. 6, 27-34 (1982); Eighth Eur. Conf. Earthquake Engng Vol. 2. 4.1.63-4.1.66 (1986).

\section{Does the $\mathrm{T}$-cell receptor bind to the MHC?}

SIR-I would like to point out one very important consequence of the model proposed by Schwartz ${ }^{1}$ and Guillet et al. ${ }^{2}$ of the molecular interactions between major histocompatibility complex (MHC) molecules and antigen. The present model postulates that the $\mathrm{T}$-cell receptor interacts with both antigen and $\mathrm{MHC}$ molecules. There is now strong experimental evidence for the interaction between antigen and $\mathrm{MHC}$, but evidence for interaction between receptor and $\mathrm{MHC}$ is circumstantial. The idea stems from the classical concept of dual recognition, itself derived from the phenomenon of $\mathrm{T}$-cell restriction. This phenomenon does not itself require that a direct interaction between receptor and (presumably)

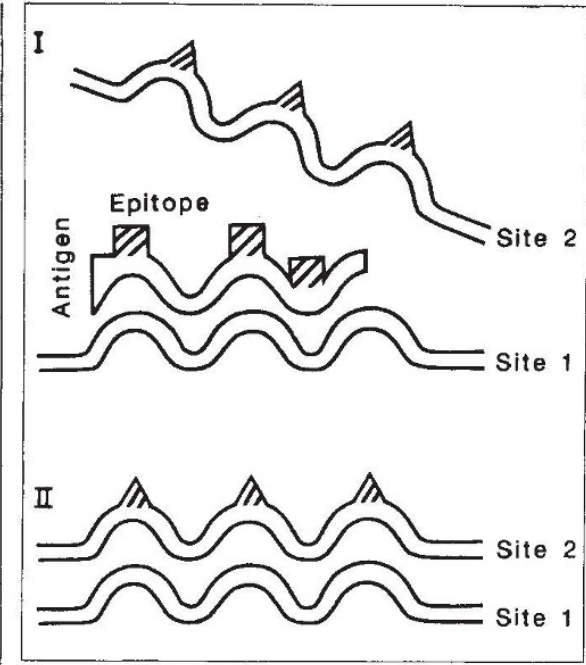

The two configurations of the MHC molecule.

polymorphic elements of the MHC exists, as a particular $\mathrm{MHC}$ molecule may select a unique portion of antigen, imposing a unique configuration on it ${ }^{3}$. The form of the epitope presented to T cells will therefore be different for different MHC molecules and this will result in the $\mathrm{T}$ cells being restricted to one $\mathrm{MHC}$ molecule. But if we take this view of restriction, it is very difficult to see how the peripheral Tcell pool can develop a repertoire which preferentially recognizes the MHC, either 'self' or foreign, without directly interacting with it. The evidence for such a skewed repertoire is now strong, if not conclusive (see ref. 4 for review).

The model proposed by Schwartz and his colleagues offers an easy solution to this problem. As shown in the figure, the MHC molecule exists in two configurations: in one the a and b chains (of Class II molecules) are prised apart, and an antigen binds to site 1 , where it is recognized by a $T$ cell. In the other configuration, the antigen is replaced by a site 2 of the MHC molecule. The crucial feature is that antigen and site 2 show molecular similarities, perhaps reflecting the fact that both bind to site 1 . T-cell receptor recognition of self-MHC plus antigen then results from recognition of those features of an antigen that mimic the self-MHC molecule. To explain why the development of the repertoire is dictated by the MHC of self, I suggest that only those $\mathrm{T}$ cells which recognize key polymorphic features on site 2 of the MHC (in closed position, shaded area in part II of the figure) are selected. To bind and trigger the T-cell receptor, an antigen bound to site 1 will have to share some structural features with site 2 , with differences constituting the T-cell epitope which presumably increase the affinity of the antigen/receptor interaction enough to result in $\mathrm{T}$ cell activation. In this model, as opposed to that of Schwartz, the similarities between antigen and site 2 are dictated both by the 